\title{
Actitudes de negación, reconocimiento, compartencia y de aprendizaje interlingüístico e intercultural en la Escuela Normal Bilingüe e Intercultural de Oaxaca (ENBIO)
}

\section{Attitudes of Denial, Recognition, Sharing, Interlinguistic and Intercultural Learning at the Escuela Normal Bilingüe e Intercultural de Oaxaca (ENBIO)}

\author{
Hugo Pacheco Sánchez \\ Escuela Normal Bilingüe e Intercultural de Oaxaca (enbio) \\ pachecomente@hotmail.com
}

8

\begin{abstract}
RESUMEN
La realidad interlingüística e intercultural que se vive en la Escuela Normal Bilingüe e Intercultural de Oaxaca (ENBIO), en México, se aprecia en la diversidad lingüística y cultural coe-xistente, situación que propicia un acercamiento a los contextos de formación (ENBIO) comunidad, escuela y aula de prácticas) a través de relatos autobiográficos de los estudiantes indígenas en formación. En éstos comparten sus experiencias respecto al contacto con ha-blantes de lenguas originarias y variantes distintas a las de su origen, con el objetivo de ubicar el desarrollo de actitudes interlingüísticas e interculturales en los procesos de formación. Al-gunos de los resultados muestran que, inicialmente, los estudiantes pasan de la negación de sus lenguas al reconocimiento y aceptación de las mismas. A su vez, cuando las condiciones son favorables, los jóvenes establecen procesos de compartencia interlingüística, práctica pro-pia de los pueblos originarios, en la cual, de manera recíproca, intercambian la "palabra", ac-ción que permite procesos de aprendizaje de otras lenguas originarias y variantes dialectales.
\end{abstract}

Palabras clave: Actitudes; negación; reconocimiento; compartencia y aprendizaje interlingüístico.

\section{Abstract}

The interlinguistic and intercultural reality experienced at Escuela Normal Bilingüe e Intercultu-ral de Oaxaca (ENBIO) Mexico, is appreciated in the coexisting linguistic and cultural diversity, a situation that allows having an approach to the training contexts; (ENBIO, community, school and practice room); through autobiographical narrations of the indigenous students in training, showing their experiences regarding contact with speakers of native language variants different 
than those of their origin; with the aim of locating the development of interlingual and intercultural attitudes in the training process. With this showing as results, that initially, they go from the denial of their languages to the recognition and acceptance of them. And how from a favorable attitude, young people establish processes of interlingual sharing, typical practices of native peoples, were in a reciprocal way they exchange the "word", an action that enables learning processes of other native languages and different dialectical variants.

Keywords: Attitudes; Denial; Recognition; Sharing and Interlingual Learning.

Fecha de recepción: 08/10/2019

Fecha de aceptación: 28/01/2020

\section{Introducción}

La Escuela Normal Bilingüe e Intercultural de Oaxaca (ENBIO) es una institución de nivel superior, encargada de formar docentes indígenas bilingües interculturales, cuyo propósito es profesionalizarlos para que desempeñen prácticas educativas bilingües e interculturales en las escuelas y comunidades originarias de la entidad oaxaqueña. La ENBio acoge a jóvenes indígenas provenientes de las diferentes regiones de Oaxaca, nativo-hablantes de lenguas originarias; se trata de estudiantes en formación inicial que, desde su ingreso y durante su estancia en esta institución, desarrollan aptitudes y actitudes, estableciendo relaciones interlingüísticas e interculturales ante la diversidad que se vive en este contexto formativo.

Tomemos en cuenta que en este estudio, lo interlingüístico y la interculturalidad se entienden como un proceso dialógico y de negociación, en el cual los sujetos establecen cambios de conciencia, de predisposiciones y valoraciones positivas sobre las culturas, sus lenguas y los hablantes. Tales elementos permiten "transformar las relaciones sociales, y la construcción de condiciones de estar, ser, pensar, conocer, aprender, sentir y vivir distintas" (Walsh, 2010, p. 78); es decir, propician que las personas sientan, piensen y actúen de una manera diferente.

Transformar las relaciones sociales requiere atender a los pueblos originarios, "implica la necesidad de poner en práctica no sólo políticas educativas y culturales diferenciadas para los pueblos indígenas, sino que éstas se elaboren cada vez más con su colaboración y asentimiento" (Calbó, 1997, p. 19). Por lo tanto, requiere considerar lo que sienten, piensan, creen y necesitan al interior de las comunidades, así como lo que piensan, creen y ofrecen los gobiernos en turno. La legitimación de las políticas planteadas debería partir de esta orientación.

Incorporar a los pueblos originarios al mundo moderno y a los procesos de intercambio cultural en una sociedad global exige que no sólo se les respete su autonomía y sus derechos, sino que se les reconozca con recursos para acceder a los nuevos desafíos. Es aquí donde los pueblos originarios deben participar en la definición de políticas que propongan el paradigma de la interculturalidad. "Desde esta posición, la interculturalidad se entiende como una herramienta, como un proceso y proyecto que se construye desde la gente" (Walsh, 2010, p. 78), que permite replantear las políticas educativas y de formación docente desde abajo y desde sus realidades lingüísticas y culturales.

La educación y la formación inicial no deben permanecer estáticas a los cambios sociales locales y globales, o desarticuladas de los procesos formativos y educativos. Más bien, la formación de docentes y las escuelas normales deben cambiar su imagen y su esencia; pasar de la perspectiva instrumental y técnica a una de la comprensión de la docencia y de la formación como praxis, como la acción y la libertad de los sujetos que se abren a los otros y que son autores y actores de sus actos a través de la palabra. Esto podría resignificar la formación y la docencia (Ducoing, 2014) a través del desarrollo de conocimientos, habilidades, valores y actitudes docentes manifiestos en sus actos reflexivos y conductuales.

52 - Actitudes de negación, reconocimiento, compartencia y de aprendizaje interligüistico e intercultural... Hugo Pacheco Sánchez. DIDAC 76 (2020): 51-59 
Son muchas las preocupaciones y los intereses que surgen en el ámbito de la formación inicial de docentes; uno de éstos es el acercamiento a lo que sucede en las escuelas normales interculturales bilingües. El presente trabajo de investigación nace de la identificación de una problemática, como parte de la observación realizada en la ENBiO y de la revisión de proyectos, planes y programas de estudio, en los que se plantea el desarrollo de conocimientos, habilidades, valores y actitudes respecto a las lenguas y culturas de los estudiantes. A partir de lo anterior, se visualizan una preferencia y una valoración positiva hacia la lengua materna de los estudiantes; además, se prioriza una atención curricular específica para el desarrollo de sus lenguas. En este proceso, se toma en consideración que, al egresar de la escuela normal, los docentes deberán contar con los perfiles deseables para el tratamiento de sus idiomas y culturas originarias y, a su vez, asumir el compromiso de regresar a sus comunidades de origen o áreas lingüísticas y culturales para su intervención pedagógica.

La pretensión de volver a sus comunidades o áreas lingüísticas y culturales de origen requiere de un análisis, ya que a partir de la revisión de una muestra de 35 docentes zapotecos de la Sierra Sur de Oaxaca de ocho generaciones (2000-2010), 19 de ellos se encontraban situados en comunidades de habla de diversas variantes dialectales del zapoteco, los 16 restantes en otros contextos de Oaxaca. ${ }^{1}$ Por otra parte, en cinco comunidades y escuelas primarias bilingües visitadas de cuatro regiones (Cañada, Sierra Sur, Mixteca y Sierra Norte) encontramos a nueve egresados de la ENBIO, hablantes de la lengua zapoteca con las variantes dialectales de la Sierra Sur, Norte y Valles: zoque, chinanteca, náhuatl y mazateca. De todos ellos, ocho se encuentran ubicados en contextos lingüísticos distintos a los de su origen. Se percibe entonces el problema de la desubicación lingüística de los docentes egresados de esta normal en las escuelas y comunidades. Al respecto, queda la duda de si realmente cuentan con los perfiles necesarios para enfrentar realidades lin- güísticas y culturales distintas, sobre todo de tipo actitudinal y cómo estas actitudes se desarrollan en la normal.

A partir del planteamiento problemático anterior, surge el interés y la preocupación por investigar las actitudes de los docentes en formación, con el objetivo de identificar y analizar las actitudes de los estudiantes indígenas hablantes de diversas lenguas originarias de Oaxaca en la ENBIO, frente a otras lenguas y hablantes en contacto interlingüístico e intercultural en los procesos de formación docente bilingüe intercultural. Así, el presente estudio fue orientado bajo la siguiente interrogante: ¿qué actitudes expresan los estudiantes indígenas respecto a sus lenguas y variantes frente a otras lenguas y hablantes a las de su origen, y qué influencia tienen en el desarrollo de actitudes interlingüísticas e interculturales en los procesos formativos? Sin duda, tales actitudes se reflejan en sus prácticas pedagógicas en contextos de desubicación lingüística.

\section{Antecedentes de estudio}

Respecto al fenómeno de las actitudes hacia las lenguas, existen diversos estudios en varias partes del mundo; se trata de un campo amplio que sigue siendo el punto de partida de muchos investigadores, entre ellos Lambert \& Tucker (1972), Genesee, G. Lambert \& Holobow (1986), quienes desarrollaron investigaciones en escuelas públicas de Canadá, a través de proyectos y programas centrados en el aprendizaje de una segunda lengua (francés) como parte del desarrollo bilingüe (inglés-francés).

Por otro lado, Campos (2014) se enfoca en el estudio de las actitudes hacia el catalán del futuro profesorado aragonés de educación primaria y señala que las actitudes de los futuros docentes hacia el catalán son desfavorables debido al escaso peso de esta lengua en el panorama social y educativo aragonés, con una presencia muy limitada como asignatura en la educación primaria y secundaria, así como una completa ausencia en la formación magisterial.

\footnotetext{
${ }^{1}$ Los datos de ubicación fueron tomados del directorio de personal de fin de cursos 2010-2011, proporcionado por la Dirección de Educación Indígena, del Instituto Estatal de Educación Pública de Oaxaca.
} 
A su vez, en Chile, un estudio desarrollado por Olate V. \& Henríquez (2010) muestra las actitudes lingüísticas de profesores de educación básica frente a la vigencia del mapudungun en contacto con el espańol, en comunidades mapuches dentro del programa de Educación Intercultural Bilingüe. Este estudio destaca el avance y el reconocimiento de la lengua y la cultura mapuches en el ámbito escolar.

En México, se destacan estudios desarrollados por Castillo $(2006,2007)$ sobre las actitudes que los maseualmej de Cuetzalan expresan hacia el mexicano y el español que hablan en distintas situaciones sociales y centra la discusión en la situación actual del mexicano frente al español; mientras tanto, Skrobot (2014) plantea una investigación realizada para conocer las actitudes lingüísticas que tienen los migrantes indígenas hacia las lenguas originarias y el español; además del análisis sobre el caso de dos escuelas primarias de la Ciudad de México.

En Oaxaca, Mena (1999) presenta un estudio de actitudes lingüísticas e ideologías educativas en el contexto de la educación indígena, del que los sujetos zapotecos de la Sierra Norte, Sur y Triqui expresan discursivamente juicios y argumentaciones respecto al uso y funciones de las lenguas indígenas y el español. Sin embargo, entre muchas otras investigaciones, no se visualizan estudios actitudinales centrados en las lenguas y los hablantes indígenas en los procesos de formación inicial en las escuelas normales con enfoque intercultural bilingüe.

\section{Las actitudes}

Villoro (2001) señala que el concepto de actitud ha desempeñado un papel fundamental en la psicología social, misma que ha contribuido en la investigación empírica de diversos campos. Su abordaje demuestra su operatividad científica, extendiendo el objeto, más allá del ámbito exclusivo de la psicología, a otras ciencias como la pedagogía y la sociolingüística.

Desde la psicología social, se describe y explica el comportamiento individual; desde la sociología, se buscan explicaciones en grupo o sociedades; la antropología se enfoca en los grupos culturales; la pedagogía estudia los comportamientos individuales o colectivos en los distintos ámbitos educativos; mientras tanto, la sociolingüística describe y explica las actitudes de los sujetos con relación a las lenguas, variantes y hablantes en contacto en diversos contextos y orientaciones de estudio.

Desde una perspectiva conductista, la actitud se refiere sólo a disposiciones adquiridas por individuos pertenecientes a un medio social determinado; en este sentido, la actitud tendió a definirse en función de la dirección favorable o desfavorable del individuo hacia un objeto. Al respecto, Thurstone (1932 en Villoro 2001) la definió como "la carga de afecto en favor o en contra de un objeto psicológico" (p. 44).

Por otro lado, una definición vigente, bajo una perspectiva mentalista, es la que estableció Allport W. (1935), quien consideraba la actitud como "un estado mental o neuronal de disposición (readiness), organizado mediante la experiencia, que ejerce una influencia directiva o dinámica sobre la respuesta del individuo a todos los objetos o situaciones con los que está relacionado" (p. 810). La disposición como producto de esas experiencias, es adquirida y la dirección que se establece puede ser negativa o positiva hacia el objeto.

Se considera que la estructura componencial de la actitud está integrada tridimensionalmente por las dimensiones cognitiva, afectiva y conativa. Las dos primeras, son producidas en la mente de los sujetos, donde la persona sabe lo que piensa y lo que siente; sin embargo, estos dos elementos activan los mecanismos de actuación.

Las actitudes hacia las lenguas y sus variantes dialectales con otras en contacto no sólo implican las lenguas, sino también sus hablantes. Así que las lenguas coexistentes en determinados contextos establecen un contacto entre ellas y esta integración interlingüística de varias comunidades de habla pone de manifiesto elementos culturales que pueden ser de reconocimiento o de rechazo, vulnerando la entrada o salida de los hablantes en términos comunicativos.

La noción de las actitudes interlingüísticas es entendida como estados mentales o predisposiciones positivas o negativas de carácter cognitivo, afectivo

54 - Actitudes de negación, reconocimiento, compartencia y de aprendizaje interligüistico e intercultural... Hugo Pacheco Sánchez. DIDAC 76 (2020): 51-59 


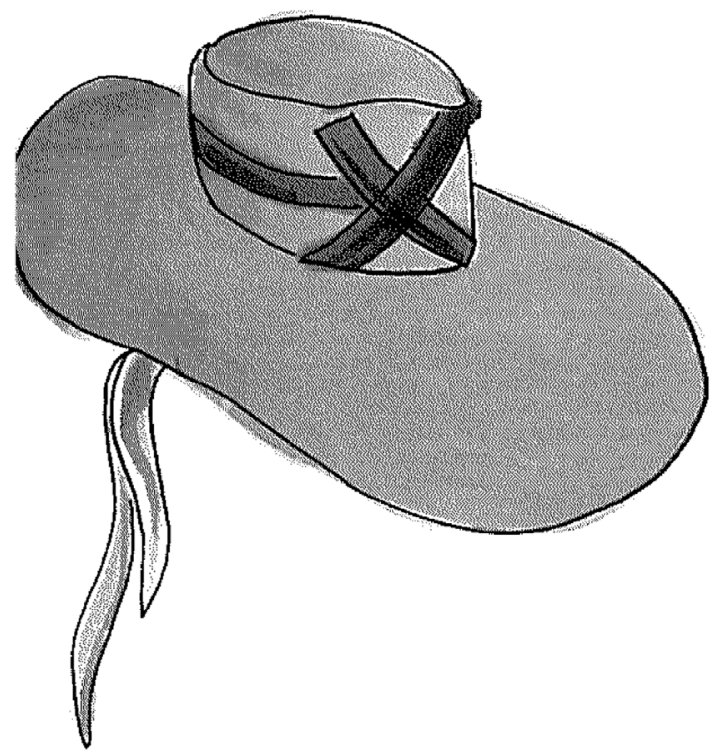

y comportamental de los sujetos hacia las diversas lenguas y variantes en contacto, así como de los hablantes en contextos multilingüísticos o de relaciones interculturales. Es decir, es la actitud asumida de un hablante de una o más lenguas hacia otro que habla distintas lenguas en un determinado contexto. Desde otra mirada, podríamos decir que se trata del sentir y el pensar hacia las diferentes lenguas y hablantes.

\section{Metodología}

Para el desarrollo de esta investigación se siguió una orientación de carácter cualitativo, a partir de "datos descriptivos: las propias palabras de las personas, habladas o escritas, y la conducta observable" (Taylor \& Bogdan, 2000, pp. 19-20), considerando la fenomenología como "un método descriptivo, filosófico, [...] capaz de dar las únicas bases seguras sobre las que se puede construir una sólida psicología empírica; [...] una filosofía universal, [...] instrumento, para la revisión metódica de todas las ciencias" (Husserl, 1997, p. 182), que permite conocer las realidades escolares y las vivencias de los sujetos en formación.

Desde la sociolingüística y la pedagogía, se utilizaron los relatos autobiográficos de experiencias interlingüísticas como técnicas de recolección de información: "una manifestación verbal y expresiva de historias lingüísticas individuales, en las cuales los sujetos enuncian una representación afectiva y explicativa del desarrollo lingüístico experimentado" (Muñoz, 2006, p. 27). Esta información permitió conocer las opiniones valorativas respecto a las lenguas y a los hablantes.

Los sujetos involucrados en el estudio son jóvenes hablantes de las lenguas chinanteca, ayuuk, mixteca, náhuatl, ombeayiüts, zoque, trique, chatina, amuzaga y zapoteca, en sus diversas variantes dialectales en la ENBIO. Entre ellos, 18 hombres y 29 mujeres, lo cual suma un total de 47 jóvenes estudiantes de tercer, quinto y séptimo semestres de la licenciatura en Educación Primaria Intercultural Bilingüe.

\section{Actitudes hacia la diversidad lingüistica}

La ENBIO es punto de encuentro, de reencuentro y de convivencia entre los hablantes de distintas lenguas. En principio, en esta escuela se gesta una posición actitudinal que empieza por el reconocimiento de la diversidad lingüística coexistente en Oaxaca, del que poco o nada se aborda en las instituciones educativas previas. Este aspecto se puede notar en el siguiente testimonio:

Antes de entrar a la escuela normal no sabía de la existencia de esta escuela y mucho menos que 
hubiera diferentes lenguas y todavía que se dividieran en variantes (H/19/M/amuzga).

Para algunos estudiantes la existencia de la ENBIO era desconocida en términos de expresión de una diversidad lingüística y cultural. Así que hasta antes de ingresar no tenían interés por la lengua y la cultura de origen, sino que fue dentro de la escuela donde se dieron cuenta del valor que representa como legado de sus ancestros:

Antes de entrar a esta normal a mí en lo particular no me interesaba saber nada sobre mi cultura o mi lengua. Fue gracias a esta normal que [se] me dio la oportunidad de saber cuál es el verdadero oro que tengo, que es la lengua que me heredaron mis antepasados (H/24/H/zapoteco).

El "antes" representa el desconocimiento de la diversidad lingüística. Una vez en la ENBIO, los estudiantes otorgan el reconocimiento de las lenguas y variantes dialectales; así que, una primera manifestación actitudinal se genera a partir de que los aspirantes se convierten en estudiantes, formando parte de la diversidad lingüística y cultural en el contexto formativo.

El hecho de tener el interés por ingresar a la ENBIO es una muestra de actitud favorable de los jóvenes. Si bien es cierto que los hablantes de lenguas originarias han sufrido discriminación, situación que los ha llevado a ocultar su uso fuera de sus contextos de origen, esto cambia una vez que ingresan a la institución, ya que allí sienten cierta seguridad de hablar sus lenguas. Se trata de un espacio que produce una empatía lingüística por hablar alguna lengua originaria; una actitud positiva que se genera a partir la convivencia y las interrelaciones comunicativas, y que permite que los estudiantes reconozcan la diversidad lingüística coexistente, produciendo en la dimensión afectiva un sentimiento de sorpresa y asombro por escuchar y saber el habla de sus compañeros de grupo. Así lo expresan los jóvenes:
Al momento de escuchar que en el estado de Oaxaca hay 16 lenguas originarias me asombró (H/01/chinanteco/2016).

Sorprendido de la diversidad y más cuando escuchaba a mis compañeros conversar en la lengua es algo que me llamó mucho la atención, debido a que yo llegué a la esta escuela sin saber de qué se trataba lo bilingüe e intercultural que lleva la normal. (H/26/H/zapoteco).

Las actitudes sobre las lenguas y los hablantes se generan al establecerse los primeros contactos interlingüísticos con sus compañeros; luego, se expresan y se desarrollan a partir de las interrelaciones que se dan entre iguales, y posteriormente, en los espacios académicos. Estas actitudes se amplían con el tratamiento y los usos de las lenguas en la institución y en los contextos de la comunidad, la escuela y el aula donde realizan sus prácticas pedagógicas intersemestrales.

\section{De la negación a la aceptación de la lengua originaria}

El hecho de sólo usar el español en los contextos educativos significa castellanizarlos y hacer a un lado las lenguas originarias, asumiendo una actitud de rechazo. Sin embargo, se da una "resistencia lingüística" (Coronado, 1995, p. 182), un sistema comunicativo que permite la sobrevivencia de las lenguas subordinadas. Estas prácticas de resistencia como lucha política se establecen a partir del desarrollo de actitudes y del uso oral o escrito de las lenguas en los contextos escolares y de formación de indígenas docentes bilingües interculturales.

La negación de pertenecer a cierto grupo, sociedad o cultura es una posición de defensa y resistencia frente a una sociedad que históricamente ha vivido en una relación asimétrica de poder, bajo situaciones de exclusión y de aniquilamiento, donde los pueblos originarios son vulnerables, ya que por ser indígenas o por hablar lenguas originarias han sido discriminados e invisibilizados.

Esta actitud social negativa hacia lo indígena motiva a que las lenguas sean desplazadas y mueran; se trata de una especie de "genocidio lingüístico". La

56 - Actitudes de negación, reconocimiento, compartencia y de aprendizaje interligüistico e intercultural... Hugo Pacheco Sánchez. DIDAC 76 (2020): 51-59 
discriminación que han sufrido los pueblos originarios durante generaciones y la violación de sus derechos lingüísticos y humanos son algunas de las causas del ocultamiento de las lenguas originarias, disminuyendo sus ámbitos de uso y pérdida de muchas de ellas (Skrobot, 2014).

La negación lingüística, como producto de la discriminación, se refleja en los discursos de los estudiantes indígenas hablantes de lenguas originarias, como parte de sus experiencias vivenciales y que son mostradas a través de sus relatos autobiográficos, donde manifiestan haber vivido discriminación lingüística hasta que ingresaron a la ENBIO. Esto se trata de un fenómeno ideológico, social e histórico que pesa sobre su conciencia y los ha orillado a asumir una actitud de negación. Al respecto algunos hablantes señalan que:

Uno vive mucha discriminación en una etapa de tu vida en la escuela, todo el tiempo piensas que los que vas conociendo son los mismos que los que te discriminaban $(\mathrm{H} / 02 / \mathrm{H} /$ chinanteco).

Muchas veces negué mi lengua originaria por miedo de que me discriminaran o que me llamaran indio o simplemente que mis amigos se alejaran de mí (H/01/chinanteco).

$\mathrm{Al}$ ingresar a la ENBIO, la negación, como defensa de la lengua originaria frente a otros sujetos que prejuiciosamente los pueden discriminar, cambia cuando entran a una etapa de reconocimiento y aceptación en el grupo. Así se puede percibir en las opiniones de los estudiantes:

Cuando me ingresé en la ENBIO todo cambió, era algo diferente y sorprendente. [...] Escuchar las conversaciones de los compañeros de otras etnias, $[\ldots]$ me dieron ganas de conversar $(\mathrm{H} / 01 /$ chinanteco).

La negación, como una actitud valorativa negativa hacia sus lenguas, cambia a partir del autorreconocimiento y la aceptación como hablantes de lenguas originarias en la ENBIO. Sin embargo, en la sociedad y en la vida cotidiana continúan las creencias del valor negativo que tienen las lenguas originarias en México, incluyendo Oaxaca.

\section{De la compartencia de lenguas al aprendizaje interlingüistico}

El aprendizaje lingüístico tiene que ver con la actitud hacia la lengua de interés que generalmente está asociada a alguna lengua extrajera. No obstante, las experiencias de los jóvenes en el contexto de la ENBIO se centran en las lenguas originarias, una actitud positiva que conlleva al aprendizaje formal y no formal de éstas, con mayor preferencia por unas que por otras.

La actitud de aceptación se da entre iguales que hablan lenguas distintas a la suya. Este cambio responde a un estado interno mediado por el contexto sociocultural; además, este nivel de aceptación y de valoración positiva de esa otra lengua lo lleva al aprendizaje a nivel léxico de una tercera lengua. En este caso, "las actitudes son características personales relativamente estables que influyen y determinan el progreso en el aprendizaje de la lengua y no al revés" (Sánchez \& Rodríguez, 1997, pp. 133-134). $\mathrm{Al}$ respecto los estudiantes manifiestan que:

1. Yo era el único chinanteco de mi variante, escuchando a mis compañeros de la etnia ayuuk, me empezó a gustar, porque siento que hablan muy bonito [...] La primera palabra que aprendí con ellos fue "uk" que significa "perro”, así empecé [...] Los compañeros nos comparten su lengua, eso es lo que más agrada [...] yo aún sigo aprendiendo ayuuk, ahora sé más palabras que antes, para mí es un gran avance y sé que voy aprender más, porque me interesa (H/01/chinanteco).

Rosa [...] es la única de mi grupo con más palabras aprendidas en las distintas lenguas, pero en especial el chatino (H/12/mixteca).

Cuando ya conocía que había diferentes variantes en la lengua zapoteca, me dio curiosidad de 
aprender algunas palabras del chinanteco, me pareció algo muy bonito [...] he aprendido algunas palabras en las lenguas de mis compañeros (H/23/M/zapoteca).

Después del reconocimiento y la aceptación lingüística, se muestran actitudes valorativas positivas hacia las lenguas y se establece un aprendizaje. En este sentido, el aprendizaje es comunitario, pues aprenden las lenguas a partir de la compartencia; es decir, "el reproducir y compartir el conocimiento originado en las comunidades entre iguales" (Martínez, 2010), concepto que Martínez utiliza como el opuesto al de competencia. La compartencia como una forma de vida de los pueblos originarios, donde las personas en reciprocidad comparten lo que tienen, lo que saben, lo que piensan y lo que sienten, unos con otros.

Lo anterior implicaría el reconocimiento y la valoración hacia las lenguas; por ende, el cambio de actitudes interlingüísticas negativas hacia positivas, enfocadas sobre las lenguas minorizadas y hacia los hablantes de éstas, permitiendo su aprendizaje a través de la compartencia.

\section{Conclusiones}

Los hallazgos del estudio ponen de manifiesto las actitudes positivas de los sujetos frente al objeto actitudinal que son las lenguas originarias y sus variantes dialectales en contextos interlingüísticos específicos. Por otra parte, se muestra que el contac- to interlingüístico genera y desarrolla actitudes positivas, que reivindican la condición étnica e identitaria para la convivencia, el respeto, la aceptación, la comunicación, la compartencia y el aprendizaje entre los sujetos de diversos pueblos originarios en la ENBIO.

Como resultado se puede apreciar un proceso de desarrollo actitudinal que va de una motivación inicial por salir adelante y ser alguien en la vida, pasando por la negación de sus lenguas como defensa frente a la discriminación y los prejuicios, hasta el reconocimiento, la importancia y el valor de sus lenguas, para luego compartirlas y en ese proceso aprenderlas a nivel léxico.

Finalmente, desde una prospectiva de la formación de los estudiantes, es importante que el indígena docente bilingüe intercultural tenga conocimientos sobre sus propias lenguas y otras existentes en su contexto inmediato; que desarrolle habilidades para establecer comunicación en su lengua y variantes; que busque estrategias comunicativas frente a otras lenguas y asuma una actitud positiva frente a la propia y las otras en contacto. Asimismo, que considere a todas las lenguas como medio de comunicación, objeto de estudio o herramienta para la enseńanza y el aprendizaje, como parte de los perfiles deseables para enfrentar realidades y situaciones de ubicación o desubicación lingüística y cultural en las escuelas y comunidades originarias.

\section{REFERENCIAS}

Allport, G. W. (1935). Attitudes. En C. A. Murchison (Ed.), Handbook of Social Psychology. Worchester, MA: Clark University Press.

Calbó Martínez, E. B. (1997). La nueva educación indígena en Iberoamérica. Educación Bilingüe Intercultural. Revista Iberoamericana de Educación, 13, 13-33.

Campos Bandres, I. O. (2014). Actitudes hacia el catalán del futuro profesorado aragonés de Educación Primaria. Sintagma, 27, 43-59.

Castillo Hernández, M. A. (2006). El estudio de las actitudes lingüísticas en el contexto sociocultural. El caso del mexicano de Cuetzalan. Anales de Antropología, 40, 283-317.

Castillo Hernández, M. A. (2007). Mismo mexicano pero diferente idioma. Identidades y actitudes lingüisticas en los maseualmej de Cuetzalan. México: INAH, UNAM.
Coronado, G. S. (1995). La resistencia lingüística como instrumento de lucha política. Anales de Antropología, 32, 179-189.

Ducoing Watty, P. (Coord.). (2014). La Escuela Normal. Una mirada desde el otro. México: IISUe, UNAM.

Genesee, F., Lambert, W. E. \& Holobow, N. (1986). La adquisición de una segunda lengua mediante inmersión: el enfoque canadiense. Infancia y Aprendizaje, 33, 27-36.

Husserl, E. (1997). Psychological and Transcendental Phenomenology and the confrontation with Heidegger (1927-1931). Dordrecht: Kluwer Academic Publishers.

Lambert, W. E. \& Tucker, G. R. (1972). The Bilingual Education of Children: The St. Lambert Experiment. Rowley: Newbury House.

Martínez Luna, J. (2010). Eso que llaman comunalidad. Pueblos originarios de Oaxaca. México: PRODICI-Conaculta.

58 - Actitudes de negación, reconocimiento, compartencia y de aprendizaje interligüistico e intercultural... Hugo Pacheco Sánchez. DIDAC 76 (2020): 51-59 
Mena Ledesma, P. (1999). Actitudes lingüísticas e ideologías educativas. Alteridades, 9, 51-70.

Muñoz Cruz, H. (2006). Cambio sociolingüístico y experiencias interculturales desde discursos autobiográficos de hablantes de lenguas indígenas. Cuadernos Interculturales, 4(07), 23-48.

Olate V., A. \& Henríquez, B. M. (2010). Actitudes lingüísticas de profesores mapuche de educación básica: vigencia y enseñanza del mapudungun en el contexto educativo. Literatura y Lingüistica, 22, 103-116. Recuperado de https:// dx.doi.org/10.4067/S0716-58112010000200008

Sánchez, M. P. \& Rodríguez, R. (1997). El bilingüismo. Bases para la intervención psicológica. Madrid: Síntesis.
Skrobot, K. (2014). Las politicas lingüisticas y las actitudes hacia las lenguas indigenas en las escuelas de México (Tesis doctoral). Universidad de Barcelona, España.

Taylor, S. J. \& Bogdan, R. (2000). Introducción a los métodos cualitativos. Espańa: Paidós.

Villoro, L. (2001). Creer, saber, conocer. México: Siglo xxi Editores.

Walsh, C. (2010). Interculturalidad crítica y educación intercultural. En Construyendo interculturalidad critica (pp. 7596). La Paz, Bolivia: Instituto Internacional de Integración del Convenio Andrés Bello.

\section{BibLIOGRAFÍA RECOMENDADA}

Cuminns, J. (2002). Lenguaje, poder y pedagogía. Madrid: Morata.

Dietz, G. \& Mateos Cortés, L. S. (2011). Interculturalidad y educación intercultural en México. Un análisis de los discursos nacionales e internacionales en su impacto en los modelos educativos mexicanos. México: SEP, CGEIB.

Fishman, J. (1982). Sociología del lenguaje. Madrid: Cátedra.
Maldonado Alvarado, B. (2016). Perspectivas de la comunalidad en los pueblos indígenas de Oaxaca. Bajo el Volcán, 15(23), 151-169.

Schön, D. A. (1998). El profesional reflexivo. Cómo piensan los profesionales cuando actúan. España: Paidós.

Walsh, C. (2017). Pedagogías decoloniales, prácticas insurgentes de resistir, (re) existir y (re)vivir. Quito: Abya Yala.

\section{SemblanZA}

Profesor en la Escuela Normal Bilingüe e Intercultural de Oaxaca (ENBIO). Maestro en Pedagogía y estudiante del doctorado en la misma disciplina en la Universidad Nacional Autónoma de México (UNAM). Ha sido promotor cultural y docente bilingüe en escuelas primarias del medio indígena de Oaxaca, así como director técnico bilingüe. Su experiencia abarca áreas administrativas de la educación, como la supervisión escolar bilingüe. Entre sus

líneas de investigación están la lengua y la cultura, así como la formación y el trabajo docente bilingüe intercultural. 